\title{
Risk factors for anaemia among HIV infected children attending care and treatment clinic at Muhimbili National Hospital in Dar es Salaam, Tanzania
}

\author{
ABEL N. MAKUBI ${ }^{1 *}$, FERDINAND MUGUSI ${ }^{2}$, PIUS M. MAGESA ${ }^{1}$, DAVID ROBERTS ${ }^{3}$ and \\ AMRANA QUARESH ${ }^{4}$ \\ ${ }^{1}$ Muhimbili University of Health and Allied Sciences, P.O Box 65001, Dar es Salaam, Tanzania \\ ${ }^{2}$ Department of Internal Medicine, Muhimbili National Hospital, Dar es Salaam,, Tanzania \\ ${ }^{3}$ National Blood Transfusion Service, Oxford University, Oxford, UK \\ ${ }^{4}$ Department of Paediatrics, Great Ormond Hospital, London, UK
}

\begin{abstract}
There is paucity of data describing the risk factors for anaemia among HIV infected children in Tanzania. This cross sectional study was carried out to determine the contributing factors for anaemia among HIV-infected children attending Muhimbili National Hospital in Dar es Salaam. Both univariate and multivariate logistic regression analyses were performed to identify possible factors associated with anaemia in HIV-infected children. A total of 75 (44\%) patients among 167 recruited HIV-infected children aged 6 months to 59 months of were found to be anaemic $(\mathrm{Hg}<11 \mathrm{~g} / \mathrm{dl})$. Multivariate logistic regression demonstrated that not being on HAART (OR 3.40, 95\%CI (1.20-9.60), having $\mathrm{CD} 4 \%<25 \%$ (OR 2.30, 95\%CI (1.20-34.60), having a history of tuberculosis (TB) (OR 3.23, 95\% CI (1.10-9.70) and having hookworm infestation (OR 5.97, 95\%CI (1.92-18.4) were independent risk factors for anaemia among HIV infected children. The analyses also showed that being HIV positive for $\geq 2.5$ years resulted into a low risk of severe anaemia compared to being HIV positive for $<2.5$ years. Taking multivitamins (OR 0.07, 95\%, CI (0.020-0.30) and antihelminthics (OR $0.27,95 \% \mathrm{CI}$ $(0.10-0.74)$ were also protective against anaemia in children. Similar factors (with exception of using antihelmintics) were associated with severe anaemia. In conclusion the factors associated with anaemia in HIV infected children were multifactorial in nature. Efforts to correct anaemia in HIV infected children should include use of HAART and treatment of infections such as TB and hookworms.
\end{abstract}

Keywords: anaemia, HIV, risk factors, children, hospital, Tanzania

\section{Introduction}

Anaemia is a common complication of HIV infection and is independently associated with disease progression and mortality (Sullivan et al., 1998; Hilgartner et al., 1991). The pathophysiology of HIV-related anaemia in childhood is not well understood and may be especially complicated amidst the dynamic changes associated with normal haematological development in early infancy. Most studies on anaemia among children in tropical Africa were conducted before the era of HIV and HAART. These studies were mainly confined to the anaemia associated with malaria, iron deficiency or with other individual factors (Calis et al., 2008). As a result, the treatment guidelines advocated by the World Health Organization deal specifically with malaria, folate deficiency, and iron deficiency, which are widely held to be the most common causes of anaemia in African children(WHO, 2010).

The aetiology of anaemia in HIV-infected patients is multifactorial in nature, with opportunistic infections, nutritional deficiencies, certain medications (including antibiotics and antiretroviral agents), and infiltrative diseases of the bone marrow are among the leading causes (Idro, 2003). Pre-existing anaemia may be worsened by any of these. Because

\footnotetext{
* Correspondence: Dr Abel Makubi; Email. makubi55@gmail.com, Mobile +255-713-307170
} 
a combination of environmental and host factors may predispose people in Tanzania living with HIV to developing anaemia, it is important to identify the various risks factors that may be implicated in this condition in order to prevent or retard its occurrence and ameliorate its severity. The objective of this study was to determine the risk factors for anaemia among HIV-children at Muhimbili National Hospital in Tanzania. Identification of these factors would also assist in development of effective strategies for controlling anaemia.

\section{Materials and Methods}

\section{Stud design and subjects}

This was a hospital based cross sectional study conducted in a HIV out-patient paediatric care and treatment clinic at the Muhimbili National Hospital (MNH) in Dar es Salaam, Tanzania. MNH is national referral hospital and university teaching hospital with 1,500 bed facility, attending 1,000 to 1,200 out-patients per week and admitting 1,000 to 1,200 inpatients per day. The hospital runs a paediatric HIV clinic from Monday to Friday. The hospital receives HIV infected children from the vicinity of hospital and from other referral hospitals across the country. The target population for the study included all consecutive HIV infected children attending the HIV clinic from September 1, 2009 to January 31, 2010. The subjects included for this study were of 6 to 59 months of age.

\section{Data collection}

The following information was collected: a child's name, file number, age, sex, residence and ethnicity; the guardian/parent level of education and occupation. The history included the status for HAART, cotrimoxazole (TMP-SMX) therapy and a history of tuberculosis (TB) disease in the previous six months before the study. Other information included a current history of chronic diarrhoea (for two weeks or more), being malnourished as documented in the file, recurrent fevers (one episode every month), recurrent malarial attacks (every month) and being treated for helminthiasis in the past three months as per parent's/guardian's ability to recall on interview. Each study subject had a physical examination on anthropometric measurements, clinical WHO stage, and presence of pallor, jaundice, lymphadenopathy ( $>2 \mathrm{~cm}$ length), hepatomegaly and splenomegaly.

The following tests were requested for each study subject: full blood picture, peripheral blood smear, CD4 level, stool for ova or cyst and blood smear for malaria. Blood for haematological tests was collected from the study subjects using sterile vacutainers with EDTA anticoagulants. A total of $5 \mathrm{mls}$ of venous blood was collected from each child from the cephalic/medial vein or femoral vein (depending on the accessibility to the vein) by the research physician. The blood sample was utilized for a full blood picture and a blood film. Full blood picture was done using a CBC Coulter Counter (Coulter Corp, Miami, FL). The blood films were stained using Leishman stain and reported by an experienced haematologist who identified the blood cell morphology and presence of malaria parasites. The CD4 count was analyzed using FACS Caliber-BD machine. Kato technique and filter method was used to examine stool for ova/cyst.

\section{Data analysis}

The data were entered into a computer and cleaned using the SPSS package software Version 15.0. Both univariate and multivariate logistic regression analyses were performed to identify possible risk factors associated with anaemia $(\mathrm{Hb}<11 \mathrm{~g} / \mathrm{dl})$ in HIV-infected 
children. Sub-analysis of factors associated with severe anaemia $(\mathrm{Hb}<5 \mathrm{~g} / \mathrm{dl})$ was also done. Adjusted and unadjusted odds ratios (OR) were calculated to find out the strength of association. The final models for factors strongly associated with either anaemia or severe anaemia was derived by forward likelihood ratio method. Anaemia was defined as a haemoglobin level of $<11 \mathrm{~g} / \mathrm{dl}$. Severe anaemia was defined as haemoglobin level $<5 \mathrm{~g} / \mathrm{dl}$ (Paul et al., 2001). Variables with P-values of $<0.05$ were considered significant.

\section{Ethical considerations}

Written consent for interview was obtained from the patient's guardians/parents. Institutional ethical clearance was obtained from the Muhimbili University of Health and Allied Sciences Ethical Committee. Patients with anaemia were managed by the investigator in consultation with the paediatrician on duty. Children whose patients/guardians did not consent were excluded for this study.

\section{Results}

\section{Social demographic and clinical characteristics of study subjects}

A total of $167 \mathrm{HIV}$ infected children were recruited for the study. Two children were excluded because the parents declined to participate in this study. The mean age of the study subjects was 3.7 years (range $=0.6-4.9$ years). The majority of the study subjects were males $(53.9 \%)$. The majority $(64.7 \%)$ of the children had mothers/guardians who were unemployed but had some formal education (86.8\%). Two thirds of the study population was on HAART at the time of recruitment. Similarly the majority of the study subjects was in the WHO clinical stage 3 or 4 and had a CD4\% level of $<25 \%$ at the time of the study. The prevalence of individual infections was $24 \%$ for malaria $25 \%$ for hookworms. Nineteen percent had a past history of TB. Twelve percent had co-infection of malaria+ hookworms while $10 \%$ had co-infections of malaria+ hookworms +history of TB.

\section{Risk factors for anaemia in HIV infected children}

A total of $75(44 \%)$ patients among $167 \mathrm{HIV}$ were found to be anaemic $(\mathrm{Hg}<11 \mathrm{~g} / \mathrm{dl}))$. Twentyfive $(15 \%)$ of the children had severe anaemia $(\mathrm{Hb}<5 \mathrm{~g} / \mathrm{dl})$. The risk of being anaemic between male and female children was similar. The level of maternal education had no relationship with the risk of having an anaemic child. Children on ZDV and Non-ZDV regimen were equally at risk of being anaemic. The risk of being anaemic was associated with the duration of HIV positivity, HAART status, WHO clinical stage, level of CD4 percent and use of multivitamins (Table 1).

In a univariate analysis, not being on HAART, having advanced WHO stage, CD4\% of $<25 \%$, a history of TB disease in the past six months at the time of study, chronic diarrhoea (14 days or more), malnutrition or recurrent malarial attacks (every month), and hookworm infestation were all associated with high risk of anaemia (Table 2). Study subjects who were $>2.7$ years of age had a lower risk of developing anaemia than those who were $\leq 2.7$ years. Likewise, being HIV positive for $\geq 2.5$ years resulted into a low risk of anaemia compared to being HIV positive for $<2.5$ years. The use of antihelmintics in three months prior to the study and taking multivitamins were found to be protective against anaemia in HIV children. 
Table 1: Univariate analysis of demographic and clinical factors associated with anaemia $(<11 \mathrm{~g} / \mathrm{dl})$ in HIV infected children

\begin{tabular}{llll}
\hline Variable & Response & \multicolumn{2}{l}{ Univariate anaemic children } \\
OR(95\%CI)
\end{tabular}

${ }^{*}=$ P-value $<0.05$

The final model derived by forward step multivariate logistic regression (Table 3) demonstrated that not being on HAART (OR 3.4, 95\% CI (1.20-9.60), having a history of TB disease in the past six months at the time of study (OR 3.23, 95\% CI (1.10-9.70), CD4 < 25\% (OR 2.3, 95\%CI (1.2-36.4) and hookworm infestation (OR 5.97, 95\% CI (1.92-18.4) were independent risk factors for anaemia among HIV infected children. Also, being HIV positive for $\geq 2.5$ years resulted into a low risk of anaemia (OR 0.12, 95\% CI (0.04-0.36) compared to being HIV positive for < 2.5 years .Taking multivitamins (OR 0.07, 95\%, CI (0.02-0.30) and antihelmintics (OR $0.27,95 \% \mathrm{CI}(0.10-0.74)$ were protective factors against anaemia. 
Table 2: Univariate analysis of co-infections and nutritional factors associated with anaemia $(\mathrm{Hb}<11 \mathrm{~g} / \mathrm{dl})$ in HIV infected children

\begin{tabular}{|c|c|c|c|}
\hline Variable & Response & Adjusted OR & $95 \% \mathrm{CI}$ \\
\hline \multirow[t]{2}{*}{ History of TB disease } & $\mathrm{No}$ & $52 / 135(38.5)$ & 1 \\
\hline & Yes & $23 / 32(71.9)$ & $4.0(1.7-9.4)^{*}$ \\
\hline \multirow{2}{*}{$\begin{array}{l}\text { History } \\
\text { diarrhoea }\end{array}$} & No & $48 / 128(37.5)$ & 1 \\
\hline & Yes & $27 / 39(69.2)$ & $3.7(1.7-8.0)^{*}$ \\
\hline \multirow[t]{2}{*}{ History of recurrent fever } & No & $26 / 65(40.0)$ & 1 \\
\hline & Yes & $49 / 102(48.0)$ & $1.3(0.7-2.6)$ \\
\hline \multirow[t]{2}{*}{ History of recurrent malaria } & No & $53 / 137(38.7)$ & 1 \\
\hline & Yes & $22 / 30(73.3)$ & $4.3(1.8-10.5)^{*}$ \\
\hline \multirow[t]{2}{*}{ Current Malaria } & No & $39 / 124(31.5)$ & 1 \\
\hline & Yes & $36 / 43(63.7)$ & $11(4.5-27.4)^{*}$ \\
\hline \multirow[t]{2}{*}{ History of malnutrition } & No & $52 / 135(38.50$ & 1 \\
\hline & $\mathrm{Ye}$ & $23 / 32(71.9)$ & $4.0(1.7-9.4)^{*}$ \\
\hline \multirow[t]{2}{*}{ Hookworm infestation } & No & $39 / 124(31.5)$ & 1 \\
\hline & Yes & $36 / 43(63.7)$ & $11(4.5-27.4)^{*}$ \\
\hline \multirow[t]{2}{*}{ Malaria + Hookworms } & No & $60 / 146(41.1)$ & 1 \\
\hline & Yes & $15 / 21(71.4)$ & $3.5(1.3-9.7)^{*}$ \\
\hline \multirow[t]{2}{*}{ Malaria+Hookworms+TB } & No & $63 / 150(42.0)$ & 1 \\
\hline & Yes & $12 / 17(70.6)$ & $3.3(1.2-9.9)^{*}$ \\
\hline
\end{tabular}

Table 3: Multivariate analysis of risk factors for anaemia $(\mathrm{Hb}<11 \mathrm{~g} / \mathrm{dl})$ in $\mathrm{HIV}$ infected children

\begin{tabular}{llll}
\hline Variable & Adjusted OR & $\mathbf{9 5 \%} \mathbf{C I}$ & P-value \\
\hline Not being on HAART & 3.4 & $1.20-9.60$ & 0.025 \\
Having history of TB & 3.20 & $1.10-9.4$ & 0.032 \\
Having hookworm & 5.78 & $1.72-19.4$ & 0.004 \\
Being HIV+ve for $\geq 2.5$ years & 0.10 & $0.02-0.76$ & $<0.001$ \\
CD4\% $<25 \%$ & 2.10 & $1.2-39.4$ & 0.025 \\
Taking anthelmintics & 0.16 & $0.10-0.94$ & 0.020 \\
Taking multivitamins & 0.09 & $0.02-07$ & $<0.001$ \\
\hline
\end{tabular}

Multivariate analysis revealed that, having a history of TB disease in the past six months at the time of study, CD4 $<25 \%$, and hookworm infestation were independent risk factors for severe anaemia among HIV infected children. In addition, being HIV positive for $\geq 2.5$ years resulted into a low risk of severe anaemia compared to being HIV positive for $<2.5$ years. Taking multivitamins was consistently a protective factor against severe anaemia. There was lack of association (with statistical significance) between gender, maternal occupation and education, type of HAART regimen, history of recurrent fevers and use of cotrimoxazole with mild and severe anaemia in HIV infected children (Table 4).

Table 4: Multivariate analysis of risk factors for severe anaemia $(\mathrm{Hb}<5 \mathrm{~g} / \mathrm{dl})$ in $\mathrm{HIV}$ infected children

\begin{tabular}{llll}
\hline Variable & Adjusted OR & $\mathbf{9 5 \% C I}$ & P-value \\
\hline Having history of TB & 5.1 & $1.10-29.7$ & 0.022 \\
CD4\% $<25 \%$ & 3.4 & $7.1-25.9$ & $<0.001$ \\
Having hookworm & 11.2 & $2.20-68.1$ & 0.004 \\
Being HIV+ for $\geq 2.5$ years & 0.11 & $0.06-0.9$ & 0.01 \\
Taking multivitamins & 0.17 & $0.02-0.9$ & $<0.020$ \\
\hline
\end{tabular}




\section{Discussion}

The study aimed to provide baseline data on the current status of risk factors for anaemia in HIV infected children at a national and referral hospital. To our knowledge, this is the first hospital based study undertaken on risk factors for anaemia in children infected with HIV in Tanzania. The study subjects were aged between 0.5 to 4.9 years with an overall mean age of 3.8 years. This created a homogenous study population in which there were no marked differences in age structure to influence the interpretation of the haematological findings related to physiological variation with age on blood cell counts and red blood cell indices. Normally at the age of six months to six years, these haematological parameters tend to remain constant (Lewis et al., 2001).

In this study despite the fact that about two thirds of the study population were already on highly active antiretroviral therapy, the majority of them were in WHO clinical stage 3 and 4 and had a CD4\% level of $<25 \%$ at the time of this study. This meant that most of the patients were in intermediate to advanced stages of immunosuppression despite being on ARV which could be expected to boost the immunity and the clinical stage. The majority of the study subjects looked clinically better than their CD4 \% level would indicate. This discrepancy might be partly explained by a possible short duration of HAART for many of the study subjects to allow significant increment in clinical status and CD4 level at the time of this study. The other possible explanation for this lack of correlation between proportion of patients who were on HAART and their clinical stage and immunity status might be a delay to seek or access HIV service thus presenting at the hospital with severe immunosuppression which could take longer time of improvement by HAART.

This study found that having a history of tuberculosis in the past six months at the time of the study was a strong independent risk factor for both mild and severe anaemia in HIV infected children. Strong association of tuberculosis and anaemia was also reported by Subbaraman (2007) when studying anaemia in HIV infected persons in Southern India. This finding is also consistent with results of another study done in the northern part of Tanzania where anaemia was found to be common among HIV-TB co-infected persons (Ngowi et al., 2008). The aetiology of anaemia in TB is likely to be multifactorial, deriving partially from anaemia of chronic disease (associated with increased IL-6) and partly from deficiencies of nutrients such as iron, vitamin A, and selenium (Villamor et al., 2008). A recent study in Malawi has also shown that occult mycobacterium disease was highly associated with anaemia in HIV infected children (Calis et al., 2008).

In this study, hookworm infestation was found to be strongly associated with both mild and severe anaemia in HIV infected children. This indicated hookworm was common in anaemic children with HIV. A similar finding was reported by Wiwanitkit et al. (2001) in Thai who found a high prevalence of intestinal parasites among the HIV-infected patients.

The role of HAART in anaemia associated with HIV infection was shown in this study. It was demonstrated that not being on HAART was an independent risk factor for anaemia. A similar finding was reported by Feyler et al. (2002) in which the use of HAART led to the reduction in the prevalence of anaemia from 13\% in 1995 to $4 \%$ in 1999. They pointed out that the improvement of immunological and clinical status associated with the increasing efficacy of antiretroviral therapy possibly explained a large part of the reduction of the risk of anaemia.

Low CD4 counts ( $<25 \%)$ and high HIV-1 RNA levels in plasma have been associated independently with increased risk of anaemia (Volberding et al., 2004). This finding is 
consistent with the results from this study in which subjects who had CD4 levels of $<25 \%$ had a higher risk of developing severe anaemia than those with CD4 levels of $>25 \%$. However, this did not correlate with advanced WHO clinical stage whose association was not statistically significant in multivariate analysis. This may be due to the finding that though most of the patients had CD4 levels of $<25 \%$, the majority were in WHO clinical stage 3 and not stage 4 which normally correspond with CD4 levels of $<25 \%$. It was interesting to find that children who had long duration of HIV positivity were less likely to be anaemic, both in univariate and multivariate analysis. The finding is different from a number of studies which have demonstrated strong association between anaemia and long duration of HIV (Belperio et al., 2004; Calis et al., 2008). In this study the explanation for this unexpected finding could be the possibility that most of the children with a long duration of HIV, have been on HAART for a long duration which could partly protect them from anaemia and opportunistic infections. It could be also due to longer regular check on their anaemia status at the clinics allowing immediate correction compared to those children who were diagnosed HIV positive for a short duration.

Multivitamins appeared to be protective against mild anaemia and severe anaemia in HIV infected children. This is consistent with the study done in Tanzania which showed that multivitamin supplementation could improve haematological status in HIV-infected women and their children in Tanzania (Fawzi et al., 2007). Micronutrient supplements have been reported to delay HIV disease progression and reduce mortality in HIV-positive persons not receiving highly active antiretroviral therapy (Drain et al., 2007).

The use of antihelmintics was found to be protective against mild and severe anaemia among HIV infected children. This was expected since antihelmintics are recommended for community prophylaxis of children against infestation with hookworms. It has been suggested that treatment for helminthes infestation may decrease the rate of viral replication in those infected with HIV (Stephenson et al., 2001).

In this study malnutrition was associated with anaemia only on multivariate analysis. However, malnutrition contributes significantly to anaemia due to the deficiency of iron, folate and B12 as well as increased infections. In one study it was shown that nutrient supplement can correct anaemia and weight loss in HIV-infected children, though this is faster in HIV-negative undernourished children than those who are HIV positive (Simpore $e t$ al., 2005). There was lack of statistical association between chronic fever, recurrent malaria, chronic diarrhoea and WHO clinical stage 3, 4 in multivariate analysis though in univariate analysis they were all associated with anaemia.

The findings of this study were limited by other factors. The lack of association in multivariate analysis of anaemia with factors such as WHO clinical stage and malaria may be attributed by the small sample size in this study. Therefore, the absence of the statistical significance in some of the associations has to be taken cautiously. Studies on serum iron, ferritin, and total iron binding capacity, folate, cobalamin level and bone marrow could give more information on the aetiology of anaemia in HIV infected children. Even with these limitations in mind, the observed findings may still be a good reflection of a true situation and this study serves as a reference for further recommendations to improve care of HIV infected children and a step for further studies on the pathophysiology of HIV related anaemia in African children.

In conclusion tuberculosis, hookworm infestation and not being on HAART and CD4 $<25 \%$ were potential risk factors for both mild and severe anaemia. Multivitamin supplementation and use of antihelmintics appeared protective against anaemia in HIV 
infected children. We recommend that all HIV positive children should be regularly screened for anaemia and continue encouraging them to use of multivitamins. The three monthly antihelmintics should be given to all HIV infected children. HAART should be made available to all eligible HIV infected children. Efforts to correct anaemia in HIV infected children should include treatment of infections such as TB and hookworms.

\section{Acknowledgements}

We thank the support of clinicians and laboratory technicians working in the paediatric HIV acre and treatment, Muhimbili National Hospital. We thank all the patients who have contributed to these findings. HIV clinic in this study was funded in by the Government of Tanzania and the US Presidents Emergency Program for AIDS relief (PEPFAR). The first author was supported by the Germany Exchange Program for Education (DAAD) and MUHAS.

\section{Conflict of interest}

There is no conflict of interest

\section{References}

Belperio, P.S. \& Rhew, D.C. (2004) Prevalence and outcomes of anemia in individuals with human immunodeficiency virus: a systematic review of the literature. American Journal of Medicine 116 (Suppl 7A), 27S-43.

Ngowi, B., Mfinanga, S., Bruun, J. \& Morkve, O. (2008) Pulmonary tuberculosis among people living with HIV/AIDS attending care and treatment in rural northern Tanzania. BMC Public Health 30, 341-347.

Calis, J.C., Phiri, K.S., Faragher, E. B., Brabin , B.J., Bates, I. \& Cuevas L.E. (2008) Severe anemia in Malawian children. New England Journal of Medicine 358, 888-99

Drain, P.K.,Mugusi, F. \&Fawzi, W.W. (2007) Micronutrients in HIV-positive persons receiving highly active antiretroviral therapy. American Journal of Clinical Nutrition 85, 333-45.

Fawzi, W.W., Msamanga, G.I., Kupka, R., Spiegelman, D., Villamor, E., Mugusi, F., Wei R. \& Hunter, D. (2007) Multivitamin supplementation improves hematologic status in HIV-infected women and their children in Tanzania. American Journal of Clinical Nutrition 85, 1335-43.

Feyler, A., Dupon, M., Ragnaud, J.M., Mercie, P., Tchamgoue, S., Lawson-Ayayi, S., Dabis, F. \& Chene, G. (2002) Anemia, HIV infection and antiretroviral (ARV) therapy: results from an observational cohort study of 5,325 patients (Aquitaine, France, 1988-1999). International Conference on AIDS 14. Abstact No TuPeC 4755 .

Hilgartner, M. (1991) Hematologic manifestations in HIV-infected children. Journal of Pediatrics 119, S47-49.

Idro, R. (2003) Severe anemia in childhood cerebral malaria is associated with profound coma. African Health Sciences 3, 15-18.

Lewis, S.M., Bain, B.J. \& Bates, I. (2001) Practical Haematology, 10 ${ }^{\text {th }}$ edition, Churchill Livingstone. 
Paul, V,. Douglas, D. \& Sullivan, P.S. (2001). HIV Management in the HAART Era: Complications, Challenges, New Directions. Medscape CME:http://cme.medscape.com/viewarticle/412897 (accessed 12 May 2008).

Simpore, J., Zongo, F., Kabore, F., Dansou, D., Bere, A., Nikiema, J.B., Pignatelli, S., Biondi. D.M., Ruberto, G., Musumeci, S. (2005). Nutrition rehabilitation of HIV-infected and HIV-negative undernourished children utilizing spirulina. Annal of Nutrition and Metabolism 49, 373-380.

Stephenson, L.S. (2001) Optimising the benefits of anthelmintic treatment in children. Paediatric Drugs 3, 495-508.

Subbaraman, R., Devaleenal, B., Selvamuthus, P., Yepthom, T. \& Kumarasany, N. (2009) Factors associated with anemia in HIV-infected Individuals in South India. International Journal of STD \& AIDS 20, 489-492

Sullivan, P.S.H.D., Chu, S.Y., Jones, J.L. \& Ward, J.W. (1998) Epidemiology of anemia in human immunodeficiency virus (HIV)-infected persons: results from the multistate adult and adolescent spectrum of HIV disease surveillance project. Blood 91, 301-308.

Villamor, E., Mugusi, F. Urassa,W., Bosch, E., Saathoff., K. \& Fawzi, W.W. (2008) A trial of the effect of micronutrient supplementation on treatment outcome, $\mathrm{T}$ cell counts, morbidity, and mortality in adults with pulmonary tuberculosis. Journal of Infectious Disease 197, 1499-505.

Volberding, P.A., Dieterich, D., Mildvan, D., Mitsuyasu, R. \& Saag, M.(2004) Anemia in HIV infection: clinical impact and evidence-based management strategies. Clinical Infectious Disease 39, 1088-9.

WHO (1998) Guidelines for the use of iron supplements to prevent and treat iron deficiency anemia. Geneva: World Health Organization, http://www.who.int/nutrition/publications/anemia_iron_pub/en/index.html. (accessed on May 4, 2010

Wiwanitkit, V. (2001) Intestinal parasitic infections in Thai HIV-infected patients with different immunity status. BMC Gastroenterology 1, 188-193. 